\title{
Stability Analysis of a Mathematical Model for Onchocerciaisis Disease Dynamics
}

\section{BAKO, DU; AKINWANDE, NI; ENAGI, AI; KUTA, FA; ABDULRAHMAN, S}

\author{
Department of Mathematics, Federal University of Technology Minna, Nigeria \\ Email: deborah.bako@futminna.edu.ng
}

\begin{abstract}
In this work, we propose a Deterministic Mathematical Model that Combines Infectious but not Blind and Infectious Blind Compartments for Onchocerciasis Transmission and Control. Onchocerciasis is usually the term used to describe river blindness, it is a disease that causes blindness, and the second largest cause of blindness after trachoma. It mainly affects the eyes and the skin. The equilibrium states of the model are obtained. The disease free equilibrium state is analysed for stability; the condition for its stability is obtained as an inequality constraint on the parameters. Results shows that although, a $60 \%$ treatment coverage rate of infected and infectious blind individuals only is better than $80 \%$ treatment coverage rate of infected but not blind individuals only. Also, all the four control strategies reduce the effective reproduction number below unity. A $40 \%$ coverage rate of fumigation and treatment of infectious but not blind is better than a $40 \%$ coverage rate of fumigation only. It further reveals that a $30 \%$ coverage rate of fumigation and treatment of infectious blind is better than $80 \%$ coverage rate of fumigation only or fumigation and treatment of infected but not blind only. We are able to show that disease free equilibrium and endemic equilibrium exists and are both locally and globally stable, and we computed the $R_{c}$ of the model and showed that it is a parameter to test for stability, we also use the Jacobi stability technique to show that disease free equilibrium and endemic equilibrium are both locally and globally stable. The sensitivity analysis results shows that the most sensitive parameter is $\rho$ while the least sensitive is $\mu_{v}$,

(C) JASEM

https://dx.doi.org/10.4314/jasem.v21i4.6
\end{abstract}

Keywords: Onchocerciasis, Mathematical model, Equilibrium state, Deterministic, Effective reproductive number, Stability,

Onchocerciasis is usually the term used to describe river blindness, it is a disease that causes blindness, and the second largest cause of blindness after trachoma. It mainly affects the eyes and the skin. It is caused by the parasitic filarial nematode Onchocerca volvulus and is transmitted by the bites of Simulium blackflies Center for Disease Control and World Health Organisation (CDC, 2013; WHO, 1995). Onchocerciasis is often referred to as River Blindness because the blackfly laid its eggs attached to rocks and vegetation submerged in fast flowing, highly oxygenated rivers and streams where larval and pupa stages develop before transformation to the adult. This result leads to high prevalence of eye disease in villages located along fast flowing rivers where the blackfly breeds. (World Health Organization, 1995)

Despite numerous strategies employed by various national and international organization to eradicate the disease, recent surveillance data from the World Health Organization (WHO) and Onchocerciasis Control Programme (OCP) reveal that more than 17.7 million are infected globally. Onchocerciasis is also endemic in Western and Central Africa and also in Central and Southern America.

Most of the cases of blindness caused by Onchocerciasis are found in sub-Sahara Africa (outside the areas covered by Onchocerciasis Control Programme; OCP, (WHO, 1995). Although Onchocerciasis is not a disease that leads to death, there is clear evidence that blindness may cause social and economic complications which may lead to early death because sight is light. Onchocerciasis is particularly prevalent in tropical Africa and parts of tropical America; (Basáñez 2002). More recent estimates by World Health Organisation (WHO, 1995) mention more than 17.7 million infected, 500000 visually impaired and another 270000 blind. About $99 \%$ of infected persons are in Africa and $11 \%$ in Nigeria and it is more prevalence in Mubi Village in Adamawa State where about $89 \%$ of the entire village suffers one form of blindness or the other. This hyper-endemic earns the community the village of the blind. The occurrence is found in Yemen and some countries in South America. Onchocerciasis is locally transmitted in thirty countries of Africa, 13 foci in the Americas (Mexico, Guatemala, Ecuador, Colombia, Venezuela,Brazil) and in Yemen. These Countries are classified as Meso and Hyper endemic by the OCP Countries categories. In West Africa, the fear of infection is one of the major causes of migration from fertile riverine areas into sub marginal lands, which results in over cultivation and low productivity (Basáñez et al. 2006) 
During the last decade, Jimmy and Horst (2003), Basáñez and Ricardez -Esquinca (2001), Jibrin and Ibrahim (2011), Ikechukwu and Thomas (2014), Shaib et al (2015), Abdon and Rubayyi (2015) and Hugo (2013) have designed mathematical models on Onchocerciasis (river blindness). Considering the works of the afore-mention authors, the study at hand is an improvement on the cited models above in that it includes;

The latent, the infectious blind and recovered classes The treatment of actively infected individuals and blind as control parameters
Incorporating the fumigation parameter Loss of immunity after recovered.

\section{MATERIALS AND METHODS}

Model Formulation: A mathematical model for the transmission dynamics and control of Onchocerciasis was developed, improving on the existing models as explained in the literature review by incorporating the infectious but not blind and the blind compartments varying population size (birth rate not equal to death removal rate), and standard incidence.

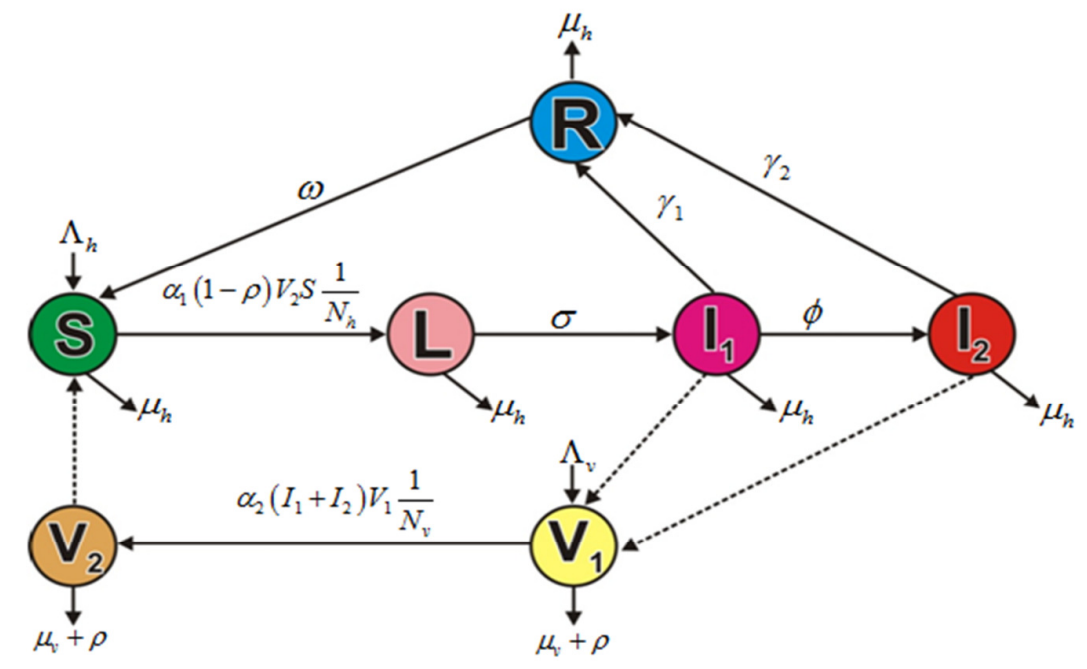

Fig 1: Schematic diagram of Onchocerciasis transmission

The model contains of the human and the vector (Black fly) populations. The human population is divided into five (5) compartments namely: the susceptible human $(S)$ latently infected human, $(L)$ Infectious but not blind human $\left(I_{1}\right)$, the Infectious Blind human $\left(I_{2}\right)$, and the Recovered human $(R)$. The Blackfly population consists of the Non- carrier blackfly $\left(V_{1}\right)$ and the carrier blackfly $\left(V_{2}\right)$.

The Susceptible human population $(S)$ is generated at a constant rate $\Lambda_{h}$ via recruitment of humans by birth and immigration into the population. It is further increased by recovered individuals losing immunity at the rate $\omega$. It is decreased by infection acquired via contact with carrier vectors at a rate $\alpha_{1}$ where $\rho($ i.e $0<\rho<1)$ is the effective fumigation rate. It is further decreased by natural death at the rate $\mu_{h}$. All the newly infected susceptible individuals moved to the latently infected compartment $(L)$.

The Latent compartment $(L)$ is decreased by progression of the disease to infectious but not blind class $I_{1}$ and natural death at the rates $\sigma$ and $\mu_{h}$ respectively. It is further decrease due to following interaction with the non-carrier blackfly $\left(V_{1}\right)$.

The infectious but not blind $\left(I_{1}\right)$ population is increased by progression of latently infected individuals at the rate $\sigma$ and decreases by complications of the infection resulting into blindness at the rate $\phi$, it further decreases by recovery due to treatment to the recovered class at the rate $\gamma_{1}$ and natural death also occurs at the rate $\mu_{h}$.

The infectious blind compartment $\left(I_{2}\right)$ is increased at the rate $\phi$ due to progression of infection from $I_{1}$ class and diminished by recovery due to treatment from Onchocerca volvulus (not the blindness) and natural death at the rate $\gamma_{2}$ and $\mu_{h}$ respectively.

The recovered compartment $(R)$ is generated from recovered individuals from the $I_{1}$ and $I_{2}$ classes at 
the rates $\gamma_{1}$ and $\gamma_{2}$ respectively. It diminishes by losing drug induced immunity and natural death at the rates $\omega$ and $\mu_{h}$ respectively.

The Non- carrier blackfly, $\left(V_{1}\right)$ are generated via birth or immigration of blackfly into the population at the rate $\Lambda_{v}$. It decreases by infection acquired when the non-carrier blackfly feed from the blood of the $I_{1}$ or the $I_{2}$ individuals at the rate $\alpha_{2}$. The blackfly

$$
\left.\begin{array}{l}
\frac{d S}{d t}=\Lambda_{h}-\frac{\alpha_{1}(1-\rho) V_{2} S}{N_{h}}+\omega R-\mu_{h} S \\
\frac{d L}{d t}=\frac{\alpha_{1}(1-\rho) V_{2} S}{N_{h}}-\left(\sigma+\mu_{h}\right) L \\
\frac{d I_{1}}{d t}=\sigma L-\left(\phi+\gamma_{1}+\mu_{h}\right) I_{1} \\
\frac{d I_{2}}{d t}=\phi I_{1}-\left(\gamma_{2}+\mu_{h}\right) I_{2} \\
\frac{d R}{d t}=\gamma_{1} I_{1}+\gamma_{2} I_{2}-\left(\omega+\mu_{h}\right) R \\
\frac{d V_{1}}{d t}=\Lambda_{v}-\frac{\alpha_{2}\left(I_{1}+I_{2}\right) V_{1}}{N_{v}}-\left(\mu_{v}+\rho\right) V_{1} \\
\frac{d V_{2}}{d t}=\frac{\alpha_{2}\left(I_{1}+I_{2}\right) V_{1}}{N_{v}}-\left(\mu_{v}+\rho\right) V_{2}
\end{array}\right\}
$$

sub-population diminishes by natural death or larvicides at the rates $\mu_{v}$ and $\rho$ respectively.

The carrier blackfly $\left(V_{2}\right)$ are generated from the Non-carrier blackfly $\left(V_{1}\right)$ that feed on infectious blood meals and similarly diminishes at the rates $\mu_{v}$ and $\rho$ respectively. Mathematical representation of the schematic diagram is given by the following ordinary differential equations.

The corresponding mathematical model equations are described by a system of Ordinary Differential Equations (ODEs) given below:

where

$$
\begin{aligned}
& N_{h}(t)=S(t)+L(t)+I_{1}(t)+I_{2}(t)+R(t) \\
& N_{v}(t)=V_{1}(t)+V_{2}(t)
\end{aligned}
$$

So that

$$
\begin{aligned}
& \frac{d N_{h}(t)}{d t}=\Lambda_{h}-\mu_{h} N_{h}(t) \\
& \frac{d N_{v}(t)}{d t}=\Lambda_{v}-\left(\mu_{v}+\rho\right) N_{v}(t)
\end{aligned}
$$

in the biological feasible region: 


$$
\left.\Omega=\left(\begin{array}{c}
S \\
L \\
I_{1} \\
I_{2} \\
R \\
V_{1} \\
V_{2}
\end{array}\right) \in \square_{+}^{7} \mid \begin{array}{l}
S \geq 0, \\
L \geq 0, \\
I_{1} \geq 0, \\
I_{2} \geq 0, \\
R \geq 0, \\
V_{1} \geq 0, \\
V_{2} \geq 0, \\
S+L+I_{1}+I_{2}+R+V_{1}+V_{2}
\end{array}\right\}
$$

Setting

$$
\begin{aligned}
& \theta=1-\rho \\
& k_{1}=\sigma+\mu_{h} \\
& k_{2}=\phi+\gamma_{1}+\mu_{h} \\
& k_{3}=\gamma_{2}+\mu_{h} \\
& k_{4}=\omega+\mu_{h} \\
& k_{5}=\mu_{v}+\rho
\end{aligned}
$$

$$
\left.\begin{array}{l}
\frac{d S}{d t}=\Lambda_{h}-\frac{\alpha_{1} \theta V_{2} S}{N_{h}}+\omega R-\mu_{h} S \\
\frac{d L}{d t}=\frac{\alpha_{1} \theta V_{2} S}{N_{h}}-k_{1} L \\
\frac{d I_{1}}{d t}=\sigma L-k_{2} I_{1} \\
\frac{d I_{2}}{d t}=\phi I_{1}-k_{3} I_{2} \\
\frac{d R}{d t}=\gamma_{1} I_{1}+\gamma_{2} I_{2}-k_{4} R \\
\frac{d V_{1}}{d t}=\Lambda_{v}-\frac{\alpha_{2}\left(I_{1}+I_{2}\right) V_{1}}{N_{v}}-k_{5} V_{1} \\
\frac{d V_{2}}{d t}=\frac{\alpha_{2}\left(I_{1}+I_{2}\right) V_{1}}{N_{v}}-k_{5} V_{2}
\end{array}\right\}
$$

\section{It can be seen that all solutions of the system (1) starting in $\Omega$}

which can be shown to be positively invariant.

\section{Effective Reproductive number $\left(R_{c}\right)$}

One of the most important concern in the analysis of epidemiological models is the determination of the asymptotic behaviour of their solution which is usually based on the stability of the associated equilibria. These models typically consist of disease free equilibrium and at least one endemic equilibrium. The local stability is determined based on a threshold parameter known as basic reproduction number $R_{c}$ this represents the average number of 
secondary cases generated by an infected individuals if introduced into a susceptible population with no immunity to the disease in the absence of interventions to control the infection. If $R_{c}<1$, then on average, an infected individual produces less than one newly infected individual over the course of its infection period. In this case, the infection may die out in the long run. Conversely, if $R_{c}>1$, each infected individual produces, on average more than one new infection, the infection will be able to spread in a population. A large value of $\left(R_{c}\right)$ may indicate the possibility of a major epidemic. Similarly, the effective reproductive number $\left(R_{c}\right)$ represents the average number of secondary cases generated by an Then,

$$
F=\left(\begin{array}{cccc}
0 & 0 & 0 & \alpha_{1} \theta \\
0 & 0 & 0 & 0 \\
0 & 0 & 0 & 0 \\
0 & \alpha_{2} & \alpha_{2} & 0
\end{array}\right)
$$

infected individual if introduced into a susceptible population where control strategies are used.

A better widely accepted and used method for finding $\left(R_{c}\right)$ that reflect its biological meaning is the next generation operator approach described by Diekmann and Heesterbeek (2000) and subsequently analysed by Van de Driessche and Watmough (2002). Using this technique we obtained the effective reproductive number, $\left(R_{c}\right)$ of the system (1) which is the spectral radius $(\rho)$ of the next generation matrix, $(K)$, $R_{C}=\rho\left(F V^{-1}\right)$.

and

$$
\begin{aligned}
& V=\left(\begin{array}{cccc}
k_{1} & 0 & 0 & 0 \\
-\sigma & k_{2} & 0 & 0 \\
0 & -\phi & k_{3} & 0 \\
0 & 0 & 0 & k_{5}
\end{array}\right) \\
& R_{c}=\sqrt{\frac{\alpha_{1} \alpha_{2}(1-\rho) \sigma\left(\left(\gamma_{2}+\mu_{h}\right)+\phi\right)}{\left(\sigma+\mu_{h}\right)\left(\phi+\gamma_{1}+\mu_{h}\right)\left(\gamma_{2}+\mu_{h}\right)\left(\mu_{v}+\rho\right)}}
\end{aligned}
$$

\section{RESULT AND DISCUSSION}

In this section, we presented some numerical simulation to monitor the dynamics of the model for various values of the associated effective reproductive number in order to confirm our analytic results on the global stability of the disease free equilibrium and the effect of different control strategies. 
Table 1: Values of Parameters of the model

\begin{tabular}{|c|c|c|c|}
\hline $\mathbf{S} / \mathbf{N}$ & Parameters & Value & Source \\
\hline 1 & $\omega$ & 0.019 & Estimated \\
\hline 2 & $\Lambda_{h}$ & $3,449,679$ & Estimated \\
\hline 3 & $\mu_{h}$ & 0.019 & CIA 2016 \\
\hline 4 & $\alpha_{1}$ & 2.12 & Shuaib 2015 \\
\hline 5 & $\alpha_{2}$ & 2.50 & Assumed \\
\hline 6 & $\mu_{v}$ & 0.00013 & WHO 2015 \\
\hline 7 & $\gamma_{1}$ & 0.067 & WHO 2014 \\
\hline 8 & $\gamma_{2}$ & 0.067 & WHO 2015 \\
\hline 9 & $\Lambda_{v}$ & $361,028.57$ & Akpan 2012 \\
\hline 10 & $N_{h}$ & $181,562,056$ & CIA 2015 \\
\hline 11 & $N_{v}$ & $2,777,142,857$ & WHO 2015 \\
\hline 12 & $\phi$ & 1.5 & Assumed \\
\hline 13 & $\sigma$ & 0.5 & Estimated \\
\hline 14 & $\rho$ & 1.0 & Assumed \\
\hline
\end{tabular}

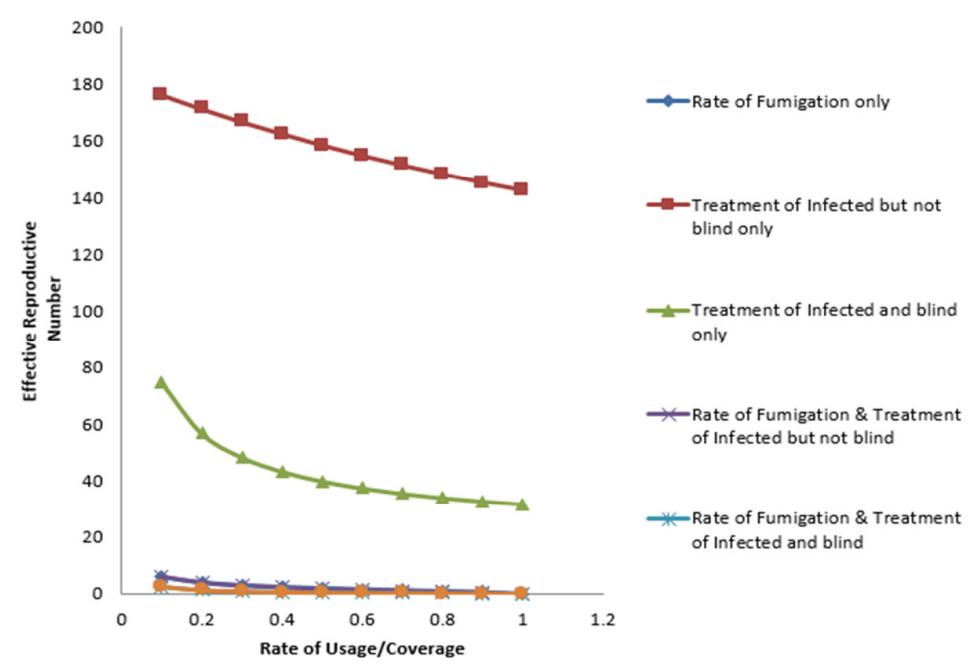

Fig 1: Comparison of the Effective Reproduction Number $\left(R_{c}\right)$ of 5 different control strategy levels. It reveals that any of the 5 control strategies have positive effects in controlling Onchocerciasis but not all can lead to stable disease free state. $\left(R_{c} \leq 1\right)$, or treatment of infectious but not blind only or treatment of infectious blind only at any coverage rate does not have much impact on the control of onchocerciasis; the disease continue to persist even though there is a decrease in morbidity. 


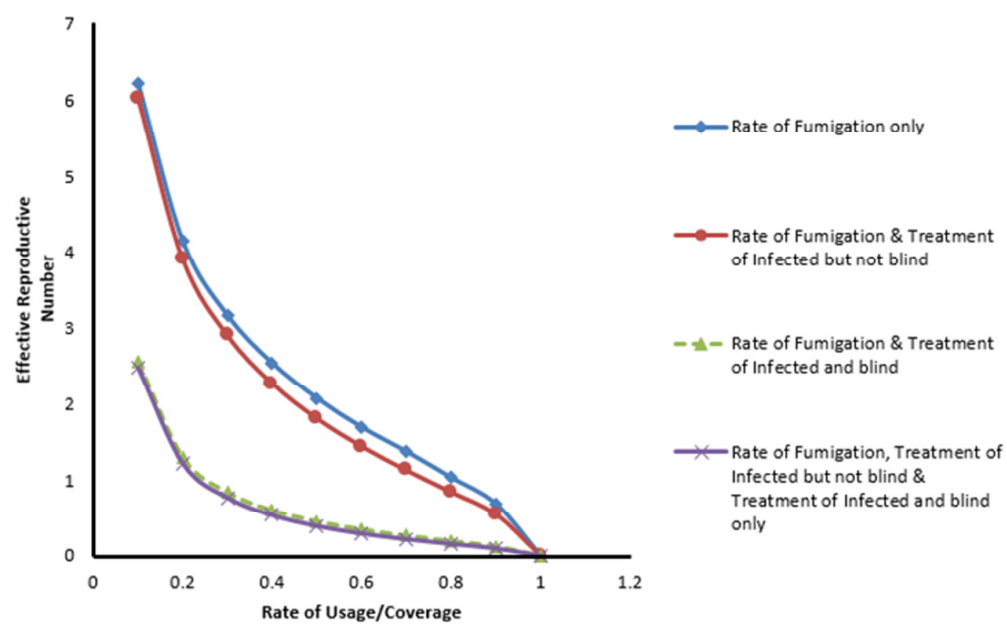

Fig 2: reveals that the four control strategies reduce the effective reproductive number below unity. Although, a $40 \%$ coverage rate of fumigation and treatment of Infectious but not blind is better than a $40 \%$ coverage rate of fumigation only. It further reveals that a $30 \%$ coverage rate of fumigation and treatment of Infectious but not blind is better than $80 \%$ coverage rate of fumigation only or fumigation and treatment of Infectious but not blind only.

Numerical Simulations: Next, we used numerical simulations to further confirm and extend the results earlier obtained.

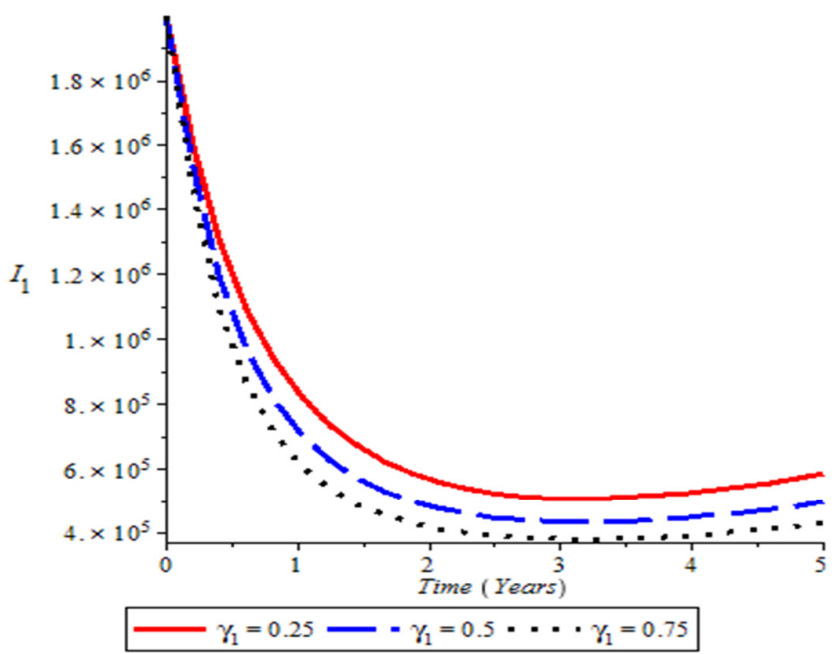

Fig 3 : A comparison between the effects of treatment of Infectious but not blind on the morbidity of $I_{1}$ individuals. Initial parameters and values used are $\gamma_{1}=0.25, \gamma_{1}=0.5$ and $\gamma_{1}=0.75$. This shows that more of the Infectious individuals should be treated with Ivermectin annually, since $75 \%$ treatment rate of Infectious but not blind reduces the morbidity to a disease free equilibrium in the first three (3) years. Hence, it is advice that $75 \%$ treatment will reduce onchocerciasis to a disease free equilibrium state. 


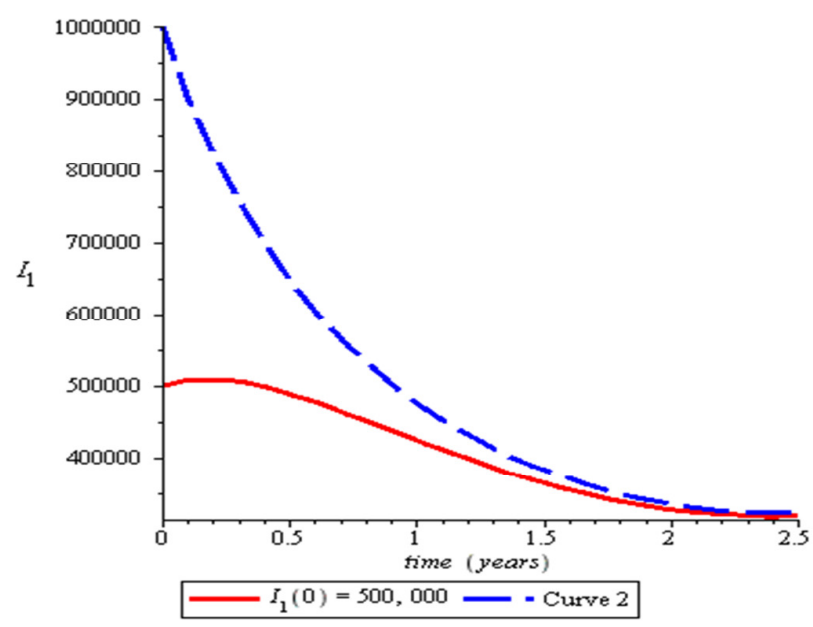

Fig 4 : the stability of Disease free equilibrium were investigated and found to be both locally and globally asymptotically stable when $\gamma_{1}=0.75, \gamma_{2}=0.75$ and $\rho=0.75$.

Conclusion: We presented a new mathematical model for the stability analysis for onchocerciasis transmission dynamics, incorporating the infectious but not blind, the infectious blind individuals and the fumigation parameter. Our analysis reveals that the four control strategies reduce the effective reproductive number below unity. Although, a40\% coverage rate of fumigation and treatment of Infectious but not blind is better than a $40 \%$ coverage rate of fumigation only. It further reveals that a $30 \%$ coverage rate of fumigation and treatment of Infectious but not blind is better than $80 \%$ coverage rate of fumigation only or fumigation and treatment of Infectious but not blind only.

\section{REFERENCES}

Abdulrahman, S (2014). A Mathematical Model for the Transmission Dynamics and Control of Hepatitis B Virus: A PhD thesis submitted to the Postgraduate School, Federal University of Technology, Minna, Nigeria.

Abdon, A; Rubayyi, TA (2015) Modelling the Spread of River Blindness Disease via the Caputofractional Derivative and Beta-derivative.

Akinwande, NI (2006). A Mathematical Model of the Dynamics of the HIV/AIDS Disease. Pandemic. Journal of the Nigeria Mathematical Society, 25, 99-108.

Akpan, SS; Alaribe, AAA; Ejezie, GC (2012). The distribution of Blackflies (Simulium Species) in Ugbem and Ukwepeyiere Communities of Biase Local Government Area of Cross River State, Nigeria.
Basáñez, M; Ricárdez-Esquinca, J (2001). Models for the population biology and control of human Onchocerciasis, Trends in Parasitology, Vol.17 No.9.

Basáñez MG, Churcher TS, Grillet ME (2009) Onchocerca-Simulium interactions and the population and evolutionary biology of Onchocerca volvulus. Adv Parasitol 263-313.

Caitlin, ED (2006). Retrospective Review of Onchocerciasis Control and Elimination Program Contributions to the Achievement of the Millennium Development Goals.

Center for Disease Control and Prevention. Onchocerciasis FAQs. May $21^{\text {st }}, 2013$

Central Intelligence Agency. (2015). World fact book for year 2012. Retrieved on October 15, 2016 from http://www.cia.gov/library/publications/theworldfactbook/ geos/ ni.html

Diekmann, O; Heesterbeek, JAP. (2000). Mathematical epidemiology of infectious Diseases: Model building, analysis and Integration. New York: John Wiley.

Hugo, CT (2013). the impact and cost of Ivermectin control strategies against River Blindness In Africa

Ibrahim, MO; Jibril, L (2011). Mathematical modelling on the CDTI prospects for Elimination of Onchocerciasis; A Deterministic approach

Ikechukwu, OC; Acho TM (2014), Mathematical modelling of the spread and control of 
Onchocerciasis in Tropical Countries: A study Nigeria.

Jimmy PO; Horst RT (2003) Competitive Dynamics in a Model for Onchocerciasis with Cross immunity.

Shaib, IO; Adeyemi. TO; Akinyemi, SG (2015) Mathematical Modelling of River Blindness with Demography using Euler Methods.

Vanden, PD; Watmough, J (2002), Reproduction Numbers and Sub-threshold Endemic Equilibria for Compartmental Models of Disease Transmission. Mathematical Biosciences, $180,29-48$
World Health Organisation (1995). Prevention of blindness and visual impairment; Priority eye disease

Hppt://www.who.int/blindness/causes/priority/en /index $3 . h t m l$

World Health Organisation (2014). Prevention of blindness and visual impairment; Priority eye disease

Hppt://www.who.int/blindness/causes/priority/en /index $3 . h t m l$ 